\title{
Construct and control of feet gait mechanisms for walking training
}

\author{
Warawut Suwalai* \\ Mechatronics Engineering Department, Faculty of Technical Education, Rajamangala University of Technology Thanyaburi, \\ Pathumthani, Thailand
}

\begin{abstract}
Gait training or gait rehabilitation is one of the major physiotherapy for stroke patients. Evidently, the robot-assisted gait training, as one part of medical technology innovation breakthrough, has important role in the rehabilitation process. The robot effectively improves treatment outcomes, fast recovery time and better convenience from traditional treatment. Typically, stroke patients are trained to walk on treadmill while a physiotherapist carefully supports and re-correct the gait pattern of the patient. For repetitive and long-time rehabilitation, it is so difficult that the physiotherapist can maintain the quality of treatment consistently. To solve such difficulties, the robotic platform is proposed for automatic guiding the gait pattern for ankle rehabilitation. The robot consists of left and right sides. Each of them is actuated by two linear and one rotational actuator. PID algorithm is implemented for position control of each joint. The experimental results show the tracking error in non-loaded and loaded cases.
\end{abstract}

\section{Introduction}

Nowadays, people suffers from the brain injury and nervous disorder. There exists some study of Thai Epidemiological Stroke [1] that discovers 1.88 percentage of the over 45-age people become stroked. Also, the number of strokes significantly increase every year.

Normally, stroke patients have problem with muscle weakness and no ability to control their legs. To recover the muscle strength and nervous system, they are properly trained by physiotherapy program until they can move by themselves or recover motor function. Typically, stroke patients are guided to walk on treadmill while a physiotherapist carefully supports and re-correct the gait pattern of the patient. However, in long-time and repetitive procedures such in physiotherapy, the quality of training is quite difficult to be maintained by human ability. To improve the quality of the physiotherapy, the robot technology is introduced to efficiently assist these procedures.

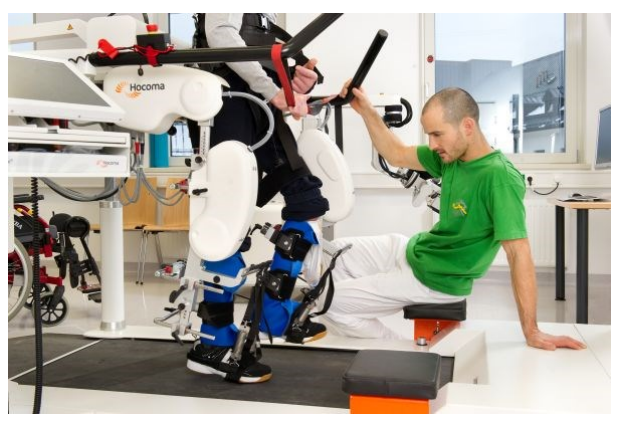

Fig. 1. Robot-assisted gait training.
Jungwon et al.[2] proposed a 6-DOF gait rehabilitation robot. The robot has footplate on a sliding device for each foot. This allows creation of the different pattern for gait training. Also, during gait training, that used the virtual environments(VEs) to guideline patient for walking procedure. Hesse et al.[3] designed a novel gait robot(G-EO-System) for repetitive practice of stroke patients based on the end-effector principle. This concept can modify the paths of the foot such as floor walking or stair climbing up and down.

In this research, the rehabilitation machine is developed for automatically assisting the stroke rehabilitation. Especially, the robot focuses on rehabilitation of the feet positions and ankle joints.

\section{Implementation}

The methodology for research compose of three topics. There are hardware design, communication system design, and programing design. The details of each topics present in this section.

\subsection{Hardware design}

\subsubsection{Design pattern}

In this research, the feet gait mechanism comprises two sides. There are left and right feet. This mechanism has the coordinate system, that use to the design for comprehensive of walking motion. The reference frame of this mechanism has $\mathrm{x}$ and $\mathrm{y}$ direction on the plane and $\mathrm{z}$ direction perpendicular to $\mathrm{x}-\mathrm{y}$ plane, respectively. Each

\footnotetext{
* Corresponding author: warawut s@rmutt.ac.th
} 
side compose the two linear motion on $\mathrm{x}$-z planar and one rotational motion on $y$-axis, that can show a threedimensional model of feet gait mechanism as figure 1 .

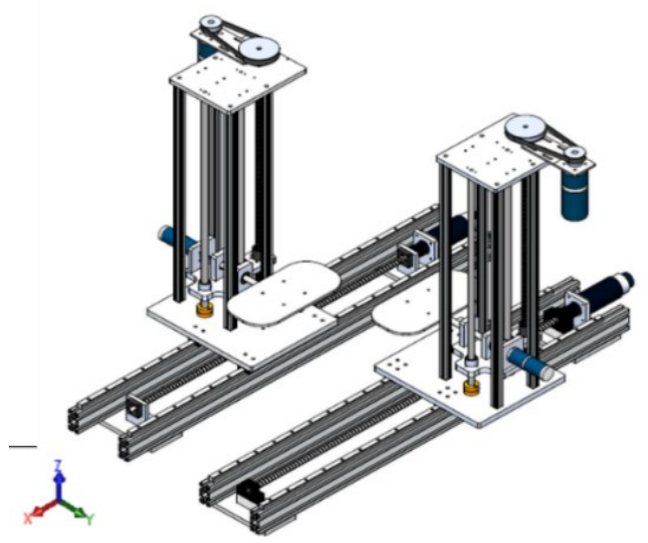

Fig. 2. 3D model and the reference frame of Feet Gait Mechanisms for Walking Training.

\subsubsection{Simulation of torque and power consumption}

Before the machine is built, the selection of mechanical parts and actuators to suit for application. It is very important. At present, we have a lot of software for designing and simulating. It is very convenient to use. Therefore, this research uses the SOLIDWORKS software to simulate torque and power consumption of actuators like a motor.

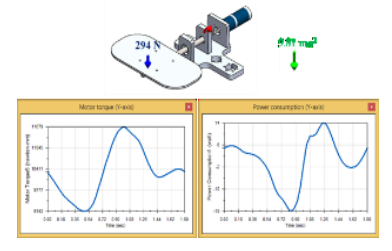

(a)

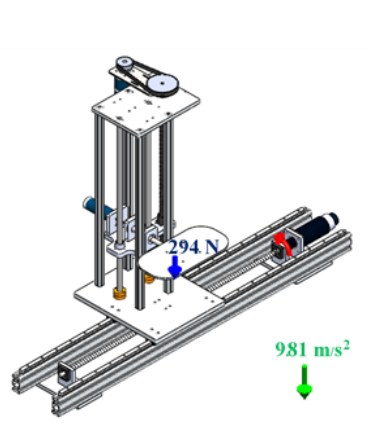

(c)

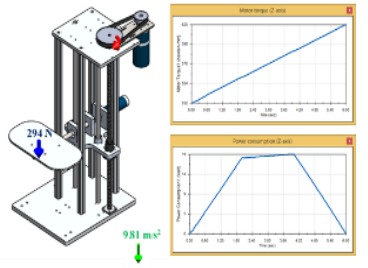

(b)

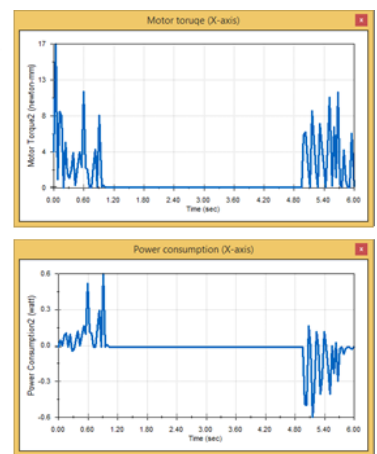

Fig. 3. Torque and power consumption results are simulated by software: rotational motion(a) linear motion(b), (c).

Firstly, we start simulation the rotational motion. Supposing situation, a weight of 30 kilograms act on the footrest. The distance of force from the rotating center is 0.035 meter. These two parameters including gravity force are set into the software. Then, the simulation results are represented by graphs. Each graph shows minimum and maximum values relative to time. The result of rotational motion simulation can be show as figure 3(a).

Secondly, we simulate the vertical linear motion. This motion include weight from rotational motion also. Total weight is sum of load and mechanical weight. The result of vertical linear motion simulation can be present as figure 3(b).

Lastly, we simulate the horizontal linear motion. This motion support total weight from rotational and vertical linear motion. The result of horizontal linear motion simulation can be show as figure 3(c). All parameters setting of two linear motion simulations are duplicated as rotational motion.

\subsubsection{Physical model}

All of feet gait mechanisms for walking training, that consists of two main parts. The first part is mechanism for movement left and right sides of foot. This mechanism force ankle position following the gait trajectory by electrical control. Mechanism are made up like 3D design that show as previous section. The second part is electrical control cabinet, that compose of electrical and electronics device such as microcontroller, motor driver, and the other electrical devices. All of actuators and sensors are connected to this cabinet. The physical model shown as figure 4.

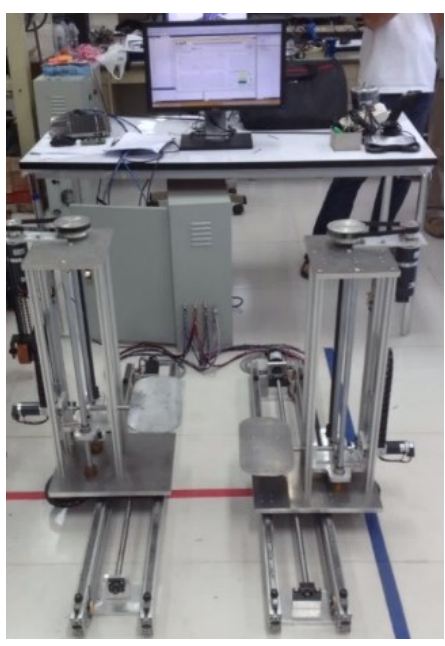

Fig. 4. Hardware of feet gait mechanisms for walking training.

\subsection{Communication system design}

The operating and monitoring of machine need to using computer. The computer communicates with the controller for transfer data between each other by using serial communication. This approach used the serial communication converter because the logic level of two devices are not completable. The logic of computer is not transistor-transistor logic(TTL) level, but logic level of microcontroller is TTL. Communication system diagram is expressed as figure 5 . 


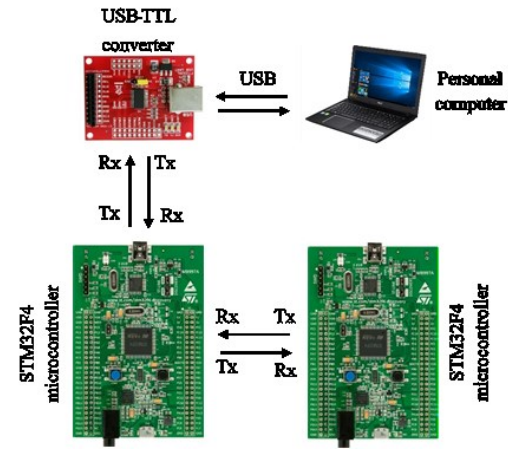

Fig. 5. Communication system connection.

\subsection{Programming design}

Software design for operation this machine, include three section program that consists of main control, user interface, and data loggers. The design procedure software preliminary used flowchart of program, that allows to guideline programming process. Flowchart of each program function expressed as figure 6 .

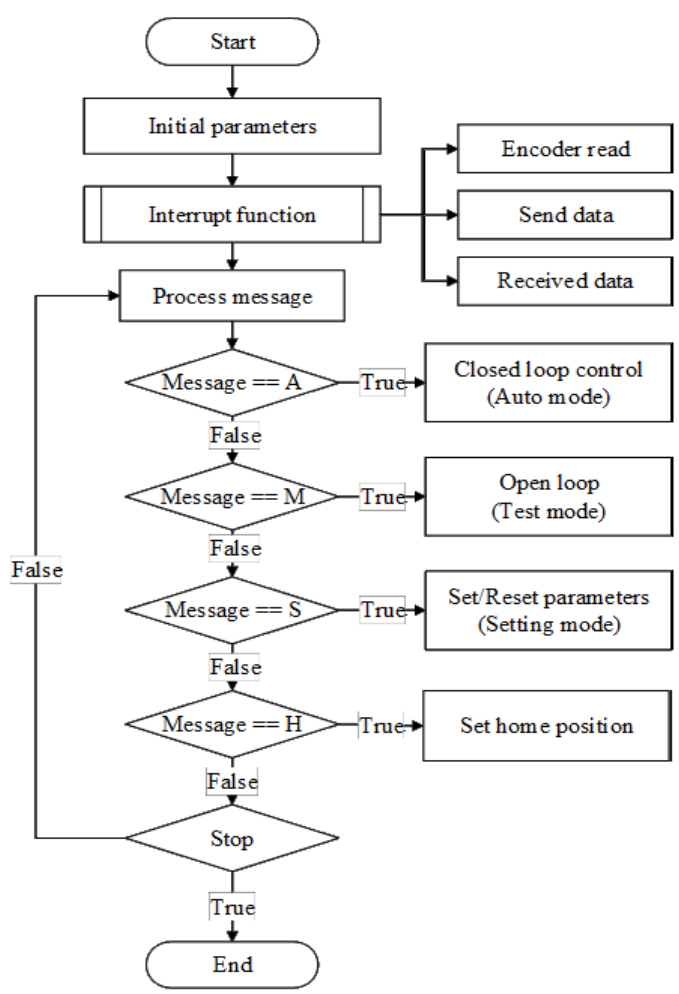

Fig. 6. Flowchart of main function in main processor.

\section{Controller Design}

For automatic control of any machine, the controller is very important for control it. The accuracy and precision of motion depend on designing the control algorithm. There are many control algorithms. In this research, The PID control algorithm is selected to control the position of each motion. The PID controller need to adjustable the gain of controller. These parameters should be suitable for system. This research consists of control the ankle joint and foot position. Therefore, every joint of the machine must be tune the gain of PID controller.

The PID controller have many methods for tuning the gain. One of methods is Ziegler-Nichols method, which is extensively used. This method is a simple and it is a trial and error tuning method based on continuous oscillations.

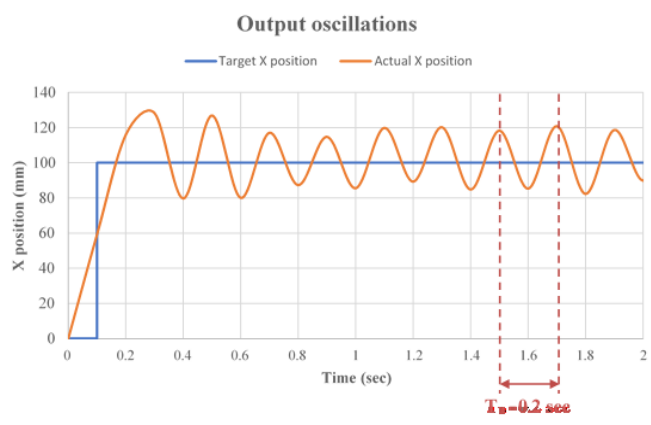

Fig. 7. Consistent oscillation and the oscillation period

For procedure to get the gain, substitution parameter which is received from figure 7 fill up into ZieglerNichols tuning rule table. The results are given as table 1 . The classical PID controller was selected in this research.

Table 1. Gain values from Ziegler-Nichols tuning method.

\begin{tabular}{|c|c|c|c|}
\hline Controller & $\mathbf{k}_{\mathbf{c}}$ & $\boldsymbol{\tau}_{\mathbf{I}}$ & $\tau_{\mathbf{D}}$ \\
\hline $\mathbf{P}$ & 75 & - & - \\
\hline $\mathbf{P I}$ & 67.5 & 0.167 & - \\
\hline $\mathbf{P D}$ & 120 & - & 0.025 \\
\hline classic PID & 90 & 0.1 & 0.025 \\
\hline
\end{tabular}

\section{Experimental results}

This section presents the experimental results of simulation of walking training. PID control algorithm is used in this task. The experiment used two scenarios, no load and weight, respectively. To investigate, that machine still perform in every suppose scenarios.

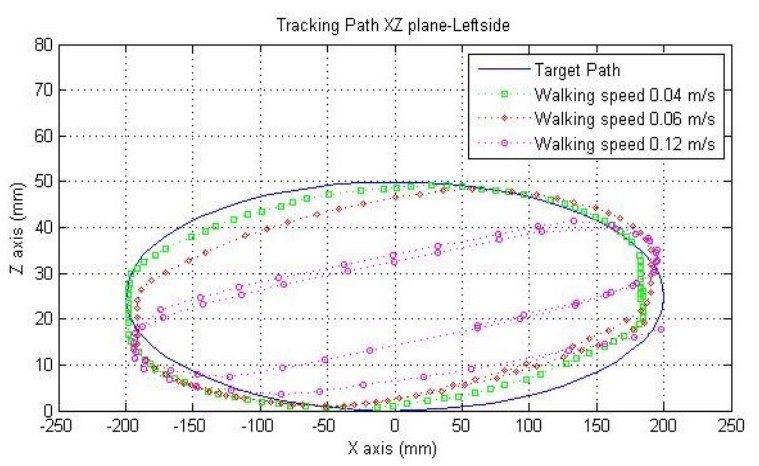

Fig. 8. Experimental results of without weight of left side foot position on $\mathrm{x}-\mathrm{z}$ plane at different walking speed. 


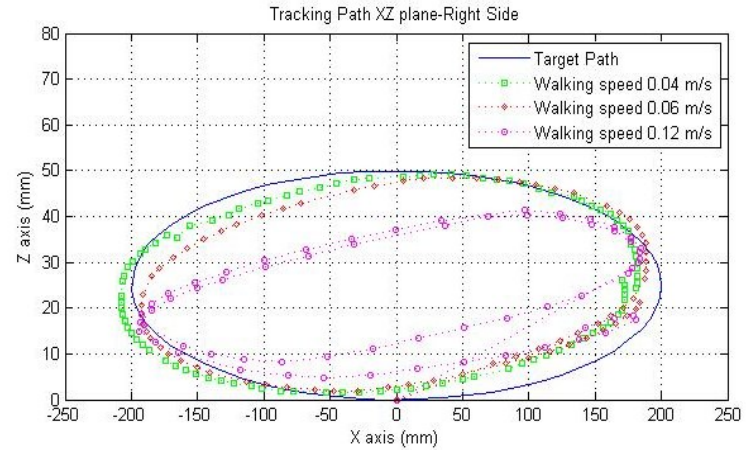

Fig. 9. Experimental results of without weight of right side foot position on $\mathrm{x}-\mathrm{Z}$ plane at different walking speed.

First test result as figure 8 and 9 that experiments are without weight by using walking speed $0.04 \mathrm{~m} / \mathrm{s}$ up to $0.12 \mathrm{~m} / \mathrm{s}$. The results of tracking path at low speed are close to the path. Increasing the speed make some error from the path, but the positions of feet are still in the pattern.

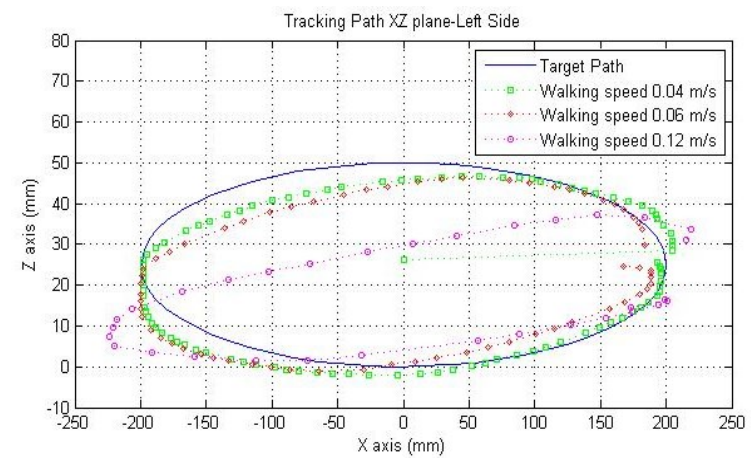

Fig. 10. Experimental results of weighted 30-kilograms of left side foot position on $\mathrm{x}-\mathrm{z}$ plane at different walking speed.

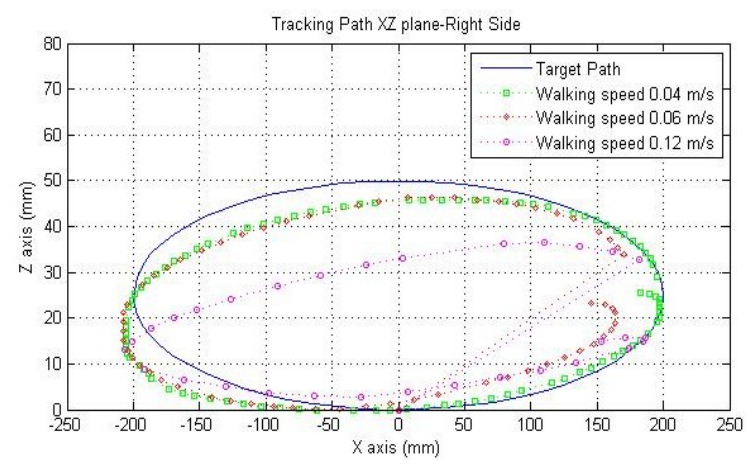

Fig. 11. Experimental results of weighted 30-kilograms of right side foot position on $\mathrm{x}-\mathrm{Z}$ plane at different walking speed.

Second Experimental result as figure 10 and 11 that experiments are with carry load 30 -kilograms by using walking speed $0.04 \mathrm{~m} / \mathrm{s}$ up to $0.12 \mathrm{~m} / \mathrm{s}$ also. The results of tracking path at low speed are close to the without weight experimentation. Increasing the speed gets large error than without weight experimentation. Some positions of the feet are in some of path form.

\section{Conclusions and recommendations}

\subsection{Conclusions}

To summarize, all the experimental results are obtained in this research. The test results are satisfactory. The mechanism can movement following the example gait pattern. Although, the position of feet is slight accuracy from the pattern when increase the speed of walking but the movement form still resembles to simulation target path. In this paper, the experimental results show only the test of feet position because the control of ankle joints is developing phase.

\subsection{Recommendations}

To Improve the position accuracy, the PID controller gain should be fine adjustment or use higher performance controller such as LQR, LQG.

\section{References}

1. NC. Suwanwela, J. Storke., 1-7 (2014)

2. J. Yoon, B. Novandy, C.H. Yoon, K.J. Park, IEEE/ASME Trans. Mechatronics., 15 no. 2, 201215 (2010)

3. S. Hesse, A. Waldner, C. Tomelleri, J. Neuroeng. Rehabil., (2010)

4. J. Yoon, J. Ryu, G. Burdea, HAPTICS 2003, 374-381 (2003)

5. W. H. Chang, Y.H. Kim, J. Storke., 15 no. 3, 174-181 (2013) 